\title{
Gao et al. (2016): Change of urinary cadmium and renal tubular protein in female works after cessation of cadmium exposure
}

\author{
Response to the Letter to the Editor by Kawada (2016)
}

\author{
Dafeng Lin ${ }^{1}$
}

Received: 2 January 2017 / Accepted: 2 January 2017 / Published online: 12 January 2017

(C) Springer-Verlag Berlin Heidelberg 2017

In the letter to the editor entitled "Change of urinary cadmium and renal tubular protein in female works after cessation of cadmium exposure", Dr. Kawada raised some concerns on our recently conducted study on urinary cadmium and renal tubular protein excretion (Gao et al. 2016). We have taken Dr. Kawada's comments into serious consideration, and would like to give our responses.

First, Dr. Kawada thought the linear regression models we used were not appropriate because of the fluctuation of urinary tubular protein excretion showed in Fig. 1b, c (Gao et al. 2016). In our opinion, although the urinary $\beta_{2}$-microglobulin $\left(\beta_{2}-\mathrm{m}\right)$ and retinol-binding protein (RBP) varied nonlinearly through the 10 years, their general and main trends were still linearly descending. Cubic curves may fit the dynamic changes of the tubular protein excretion more precisely, but they will also complicate the relationship with urinary cadmium. Linear models, on the contrary, simplified the relationship as well as yielded significant statistical results, which suggested that linear regression models were just appropriate, even if no better than nonlinear models.

As for whether the fluctuation of urinary tubular protein excretion was partly caused by creatinine adjustment, we can provide neither supportive nor opposite evidence due to lack of unadjusted data as stated in Page 1143-1144 of the paper. And based on results in the paper, urinary $\beta_{2}-\mathrm{m}$ and RBP obviously decreased in 10 years after cessation of cadmium exposure.

Dafeng Lin

david1385@foxmail.com

1 Shenzhen Prevention and Treatment Center for Occupational Diseases, Shenzhen, Guangdong, China
Second, Dr. Kawada pointed out that our study prepared no reference population of non-cadmium exposure, and there was a discrepancy of urinary $\beta_{2}$-m when compared with other reports of similar studies. Indeed, we did not include control population in the study, however, we cited urinary $\beta_{2}$-m of the local general population in Page 1143, which can be compared with that of the nickel-cadmium battery workers.

Finally, Dr. Kawada cited some results from the study by Liang et al. (2012), which suggested that urinary tubular damage in moderately and highly polluted area was progressive and not reversible after reduction of exposure. Dr. Kawada concluded that cadmium-related tubular function in female nickel-cadmium battery workers required further evaluation. We quite agree with Dr. Kawada. Contradiction has long existed on reversibility of renal function after cessation or reduction of cadmium exposure. Liang et al. even reported opposite results with the same population in one of their previously published papers (Wu et al. 2008). More studies are urgently needed to clarify the issues.

We really appreciate Dr. Kawada's comments on our study. If Dr. Kawada or other scholars have any other concerns about our study, please do not hesitate to contact us.

\section{References}

Gao Y, Zhang Y, Yi J, Zhou J, Huang X, Shi X, Xiao S, Lin D (2016) A longitudinal study on urinary cadmium and renal tubular protein excretion of nickel-cadmium battery workers after cessation of cadmium exposure. Int Arch Occup Environ Health 89:1137-1145

Liang Y, Lei L, Nilsson J, Li H, Nordberg M, Bernard A, Nordberg GF, Bergdahl IA, Jin T (2012) Renal function after reduction in cadmium exposure: an 8-year follow-up of residents in cadmium-polluted areas. Environ Health Perspect 120:223-228

Wu X, Liang Y, Jin T, Ye T, Kong Q, Wang Z, Lei L, Bergdahl IA, Nordberg GF (2008) Renal effects evolution in a Chinese population after reduction of cadmium exposure in rice. Environ Res 108:233-238 